\title{
Diversity, natural infection and blood meal sources of phlebotomine sandflies (Diptera, Psychodidae) in the western Brazilian Amazon
}

\author{
Antonio Marques Pereira Júnior ${ }^{1,2,3 /+}$, Ana Beatriz Nascimento Souza ${ }^{2}$, Thaís Santos Castro², \\ Michelli Santos da Silva' ${ }^{2}$, Paula Frassinetti Medeiros de Pauloº, \\ Gabriel Eduardo Melim Ferreira ${ }^{3,4}$, Jansen Fernandes de Medeiros ${ }^{2,3}$ \\ 'Fundação Universidade Federal de Rondônia, Programa de Pós-Graduação em Biologia Experimental, Porto Velho, RO, Brasil \\ ${ }^{2}$ Fundação Oswaldo Cruz, Laboratório de Entomologia, Porto Velho, RO, Brasil \\ ${ }^{3}$ Instituto Nacional de Epidemiologia na Amazônia Ocidental, Porto Velho, RO, Brasil \\ ${ }^{4}$ Fundação Oswaldo Cruz, Laboratório de Epidemiologia Genética, Porto Velho, RO, Brasil
}

BACKGROUND The state of Rondônia (RO) is a hot spot for human cases of cutaneous leishmaniasis. Many sandfly species in $\mathrm{RO}$ are putative vectors of leishmaniasis.

OBJECTIVES This study examines the diversity patterns and the presence of Leishmania DNA and blood meal sources of sandflies in RO.

METHODS A sandfly survey was performed between 2016 and 2018 in 10 municipalities categorised into three different environment types: (i) Conservation Unit (CUN) - comprised of preserved ombrophilous forests; (ii) Forest Edge (FE) - small forest fragments; and (iii) Peridomicile (PE) - areas around dwellings.

FINDINGS A total of 73 species were identified from 9,535 sandflies. The most abundant species were Psychodopygus davisi (1,741 individuals), Nyssomyia antunesi $(1,397)$, Trichophoromyia auraensis $(1,295)$ and Trichophoromyia ubiquitalis $(1,043)$. Diversity was the highest in CUN, followed by the FE and PE environments. One pool of Ps. davisi tested positive for Leishmania braziliensis, reinforcing the possibility that Ps. davisi acts as a vector. The cytochrome b (cytb) sequences were used to identify three blood meal sources: Bos taurus, Homo sapiens and Tamandua tetradactyla.

MAIN CONCLUSIONS Our results demonstrated that sandflies can switch between blood meal sources in differing environments. This study enhances the knowledge of the vector life cycle in RO and provides information relevant to leishmaniasis surveillance.

Key words: Leishmania - vector - reservoirs - protected areas - state of Rondônia

Phlebotomine sandflies (Diptera, Psychodidae) are small insects that act as natural vectors for protozoans of the genus Leishmania Ross (Kinetoplastida, Trypanosomatidae), which causes cutaneous leishmaniasis (CL) and visceral leishmaniasis (VL) in humans. ${ }^{(1)}$ Sandflies are distributed worldwide, but their diversity is the highest in the neotropical region, where 530 species have been recorded.(2)

Of the 280 sandfly species found in Brazil, 13 species are the proven vectors of leishmaniasis ${ }^{(3)}$ and 11 species, including Bichromomyia flaviscutellata (Mangabeira), Bichromomyia olmeca nociva (Young \& Arias), Migonemyia migonei (França), Nyssomyia anduzei (Rozeboom), Nyssomyia antunesi (Coutinho), Nyssomyia umbratilis (Ward \& Fraiha), Nyssomyia whitmani (Antunes \& Coutinho), Psychodopygus complexus (Mangabeira), Psychodopygus davisi (Root), Psychodopygus wellcomei (Fraiha, Shaw \& Lainson), and Trichophoromyia ubiquitalis (Mangabeira), might be involved in the transmission of Leishmania that cause CL.(3)

doi: 10.1590/0074-02760190170

+ Corresponding author: junior.ampj@gmail.com

(D) https://orcid.org/0000-0003-2936-1857

Received 9 May 2019

Accepted 26 June 2019
Brazil is also home to a range of putative vectors such as Nyssomyia intermedia, Nyssomyia neivai, ${ }^{(3)}$ Nyssomyia shawi, ${ }^{(4)}$ Psychodopygus carrerai, Psychodopygus hirsutus hirsutus ${ }^{(5-7)}$ and Trichophoromyia auraensis (Mangabeira). Leishmania (Leishmania) infantum Nicolle is the causative agent of VL, and its primary vector is Lutzomyia longipalpis (Lutz \& Neiva), which is distributed throughout Brazil. ${ }^{(2)}$ Other species associated with the transmission of Le. (Le.) infantum include Lutzomyia cruzi (Mangabeira) ${ }^{(8)}$ Mg. migone $i^{(9)}$ and Pintomyia fischeri. ${ }^{(10)}$

$\mathrm{CL}$ occurs in all regions of the Brazilian Amazon. Its incidence rates are the highest in the states of Pará (PA) and Amazonas (AM). ${ }^{(11)}$ In contrast, VL is endemic to the states of Mato Grosso (MT), Mato Grosso do Sul, Roraima, Tocantins and PA.(11) The zoonotic transmission risk and incidence of this disease are increased by certain factors, including (i) the presence of an ombrophilous forest, (ii) a variety of blood meal sources (e.g., tapirs, opossums, sloths and armadillos), (iii) a variety of Leishmania species and (iv) the presence of sandfly vector species..$^{(5,12,13)}$ In major transmission foci, high incidences of CL might also be the result of human exposure to the environment through activities, such as hunting and fishing, or the result of recent deforestation caused by the construction of hydroelectric power plants and roads. ${ }^{(14)}$

$\mathrm{RO}$ is located in the western Amazon basin; it borders the states of Acre (AC), AM and MT and it shares an in- 
ternational frontier with Bolivia. The state is comprised of open ombrophilous forests that have been decreasing in area since the 1970s as a result of agriculture activities. RO has the third highest incidence of CL in north Brazil, having registered approximately 12,000 cases between 2007 and 2018; according to the Brazilian Health Ministry, this incidence rate is the result of intense zoonotic transmission. ${ }^{(1)}$ No human cases of VL have been reported in RO; however, this state is thought to be at risk for VL because canine cases have recently occurred in the municipality of Cacoal. ${ }^{(15)}$ Previous studies have reported that human cases of CL in RO have been caused by the following Leishmania species: Leishmania (Leishmania) amazonensis Lainson \& Shaw, Leishmania (Viannia) braziliensis Vianna, Leishmania (Viannia) guyanensis Floch, Leishmania (Viannia) lainsoni Silveira, Ishikawa, Souza \& Lainson, Leishmania (Viannia) lindenbergi Silveira, Ishikawa \& Sousa, and Leishmania (Viannia) shawi Lainson, Braga, Souza, Póvoa \& Ishikawa. ${ }^{(16)}$

Despite the presence and high diversity of known CL vectors, such as Bi.flaviscutellata, Ny. antunesi, Ny. umbratilis and Th. ubiquitalis, ${ }^{(4,13)}$ few studies conducted in RO have demonstrated the natural infection of sandflies. $(4,7,13,17)$ The presence and high abundance of Leishmania in RO suggest that sandflies maintain their transmission cycles in the this region. ${ }^{(4,7,13,17)}$

The epidemiological knowledge of sandflies can be improved by studying their blood meal sources ${ }^{(18,19)}$ and identifying putative reservoirs. The mitochondrial genes, such as cytochrome $b$ (cytb), have been used as molecular markers for the detection of their blood meal sources. ${ }^{(18-20)}$ Thus, the present study aimed to characterise the sandfly fauna and identify their blood meal sources, as well as to assess the natural infection caused by Leishmania in RO.

\section{MATERIALS AND METHODS}

Study areas - RO is located in the northern region of Brazil; it borders AM to the north, MT to the east and $\mathrm{AC}$ to the west and shares an international border with Bolivia to the southwest (Fig. 1). It has an area of approximately $238,000 \mathrm{~km}^{2}$ and contains 52 municipalities.

The sandfly fauna was collected in three environments: Conservation Unit (CUN) - characterised by large areas of ombrophilous rain forest; Forest Edge (FE) - characterised by small forest fragments near urban areas; and Peridomicile (PE) - areas around dwellings that are situated near small forest fragments and contain enclosures where domestic animals are raised. Collections in the CUN environment were conducted between 2016 and 2017 at three places (Fig. 1): the Jaru Biological Reserve (REBIO Jaru), which has a territory that covers six municipalities (Machadinho D'Oeste, Vale do Anari, Theobroma, Ouro Preto do Oeste, Vale do Paraíso and Ji-Paraná) where collection were made in May and December of 2016 and April and July of 2017; the Jamari National Forest (FLONA Jamari), located north of RO in the municipality of Itapuã do Oeste, where collections were made in April and August of 2016 and April and October of 2017; and Guajará-Mirim State Park, located to the west of RO between the municipalities of Nova Mamoré and Guajará-Mirim, where collections were made in May and August of 2016 and April and November of 2017.

Collections in the FE and PE environments were conducted between 2016 and 2018 in the municipalities of Cacaulândia and Monte Negro (where collections were made in October 2016, June 2017 and May 2018); Cacoal, Ji-Paraná and Vilhena (where collections were made in July 2016, May 2017 and April 2018); and Guajará-Mirim and Porto Velho (where collections were made in May 2016, April 2017, and June 2018) (Fig. 1).

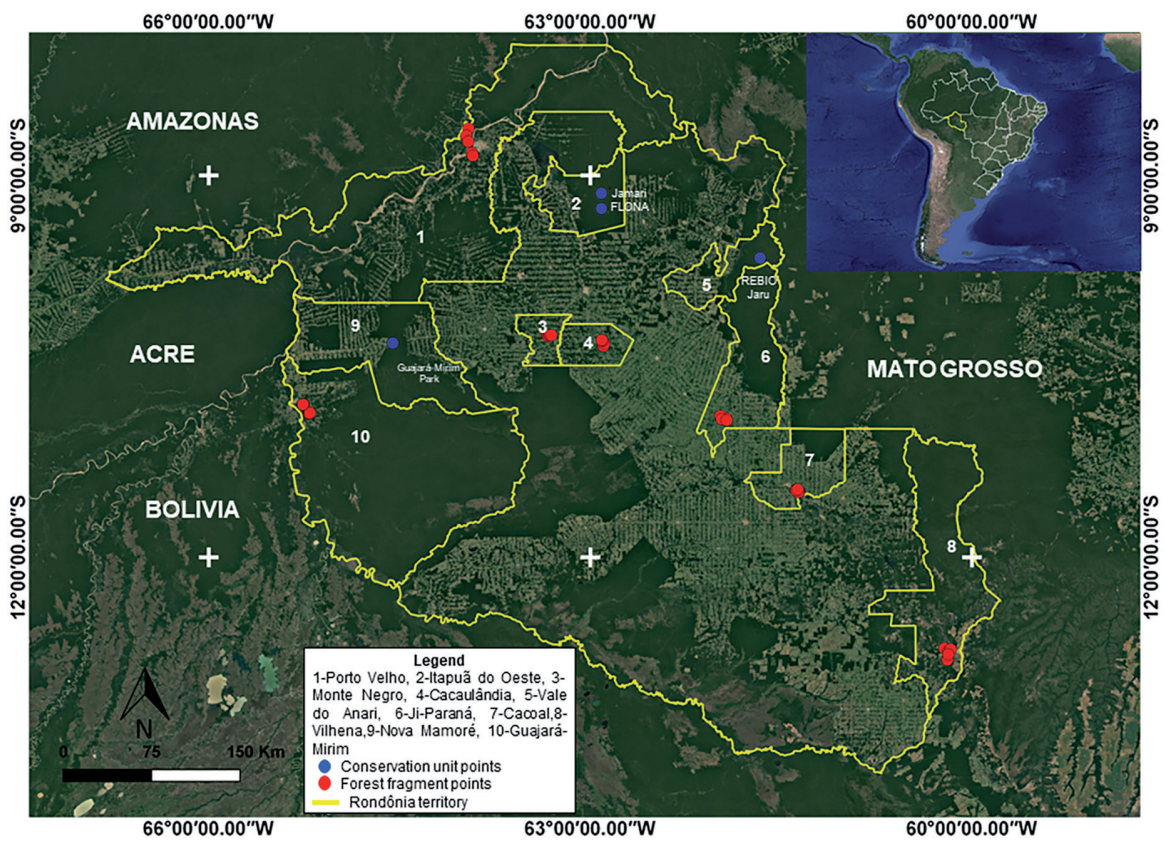

Fig. 1: sandfly collection points distributed in the state of Rondônia, Brazil. 
Sandfly collection and identification - In the CUN environments, collections were made along two different trails and sampling was performed twice in 2016 and twice in 2017. Six Hoover Pugedo (HP) light traps were set along each trail (12 HPs/reserve) and collections were made between 06:00 p.m. and 07:00 a.m. for five consecutive days.

Collections in the FE and PE environments were made from 2016 to 2018 at five locations within each municipality. At each location, one trap was set in the FE environment and two traps were set in the PE environment, using a total of 15 traps per municipality.

Male sandflies were clarified in $10 \%$ potassium hydroxide $(\mathrm{KOH})$, washed in $10 \%$ acetic acid and slidemounted in Berlese fluid. Females were divided into engorged and non-engorged specimens, and their heads and genitalia were clarified and slide-mounted as above. The thorax and abdomen of each female were stored in a microtube with $96 \%$ ethylic alcohol for further molecular analysis. Species identification was carried out using the morphological characters described by Galati. ${ }^{(2)}$

Molecular detection of Leishmania - Females were sorted according to species abundance, collection location and environment type and were separated into pools of 2-20 specimens. DNA extraction and polymerase chain reaction (PCR) assays were performed by targeting $k \mathrm{DNA}$ and hsp70, as described elsewhere. ${ }^{(4,21)}$ The Th. ubiquitalis males and the Le. amazonensis reference strain IOC/ L0575 (IFLA/BR/1967/PH8) were used as positive controls and ultrapure water was used as the negative control.

Sandfly blood meal sources - Engorged females were separated according to species, municipality, and environment type. During DNA extraction, three samples were used as negative controls: one sample containing DNA-free water and two samples containing a female sandfly with no blood present in the gut. DNA extraction was carried out using the phenol/chloroform protocol described by Sambrook and Russell. . $^{(2)}$ A PCR was carried out using the primers cytb 1 and cytb 2, which are complementary to the conserved region of the cytb gene in vertebrate mitochondrial DNA.(23)

The PCR amplification was carried out in a $50 \mu \mathrm{L}$ reaction volume containing $25 \mu \mathrm{L}$ (1X) Go Taq Colorless (Promega ${ }^{\circledR}$, Madison, WI, USA), $1.5 \mu \mathrm{L}$ of each primer (cytb 1 and cytb 2, $10 \mu \mathrm{M}$ each) and $5 \mu \mathrm{L}$ of DNA (< $250 \mathrm{ng}$ ). The amplifications were performed in a thermocycler (Veriti ${ }^{\circledR}$ - Applied Biosystems, Foster City, $\mathrm{CA}, \mathrm{USA}$ ) with an initial denaturation of $95^{\circ} \mathrm{C}$ for $5 \mathrm{~min}$, followed by 35 cycles of denaturation at $95^{\circ} \mathrm{C}$ for $30 \mathrm{~s}$, annealing at $53^{\circ} \mathrm{C}$ for $30 \mathrm{~s}$ and extension at $72^{\circ} \mathrm{C}$ for 1 min, with a final extension at $72^{\circ} \mathrm{C}$ for $6 \mathrm{~min}$. Amplified products were purified using the QIAquick Purification Kit (Qiagen, Hilden, Germany) and submitted to the Fiocruz Sequencing Facility (Rio de Janeiro, RJ, Brazil).

Data analysis - Interpolation and extrapolation curves (iNEXT) were used to evaluate sample coverage and compare diversity indexes (Shannon and Simpson) between environments. Comparisons were made using Hill numbers expressed as order q values, and the data were analysed in the $\mathrm{R}$ program. ${ }^{(7)}$
The sequences (hsp 70 and cytb) were analysed using the Phred, Phrap and Consed software programs, ${ }^{(24)}$ with the minimum value defined as $\mathrm{Q}=30$. The consensus sequences were submitted to the Basic Local Alignment Search Tool (BLAST) (http://blast.ncbi.nlm.nih.gov/ Blast.cgi) and compared with the sequences obtained from the National Center for Biotechnology Information (NCBI) GenBank database (http://www.ncbi.nlm.nih. gov/genbank/).

Ethics - The study was performed under authorisations 43702-1 and 56321-1 SISBIO/ICMBio/MMA.

\section{RESULTS}

A total of 73 species and 14 genera were identified

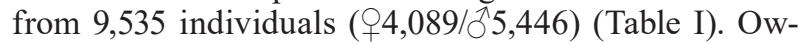
ing to the absence of morphological characters, 118 individuals were identified only at the genus level, with 49 individuals belonging to the genus Trichophoromyia and 69 to Trichopygomyia. The most abundant species were Ps. davisi (1,741 individuals), $N y$. antunesi $(1,397), T h$. auraensis $(1,295)$ and Th. ubiquitalis $(1,043)$; these four species comprised $57 \%$ of all individuals collected.

A sample coverage analysis indicated that sandfly populations were sufficiently represented in all environments. The CUN, FE and PE environments yielded $5,847,2,111$ and 1,457 individuals and 68,58 and 47 species, respectively, at $99 \%$ sample coverage (Table I).

Species diversity was the highest in the CUN environments, followed by the FE and PE environments. The CUN environments yielded the highest Shannon index $\left(H^{\prime}\right)=19.5$ common species and Simpson index $(1 / D)$ $=6.9$ dominant species, followed by FE with $\mathrm{H}^{\prime}=14.5$ common species and $1 / \mathrm{D}=6.9$ dominant species and $\mathrm{PE}$ with $\mathrm{H}^{\prime}=10.3$ common species and $1 / \mathrm{D}=5.2$ dominant species (Table I, Fig. 2).

A total of 1,755 females were divided into 274 pools representing 35 species. The PCR targeting of $k$ DNA and hsp70 identified one pool of Ps. davisi infected with Le. $($ Vi.) braziliensis (query cover $=100 \%$, identity $=$ 100\%, GenBank accession KX573933.1) (Fig. 3). The infected pool was collected from an FE environment in the municipality of Monte Negro.

Blood meal sources were identified by taking samples from 15 engorged females belonging to the following species: Bi. flaviscutellata (1), Ny. antunesi (4), Psathyromyia dendrophyla (2), Ps. carrerai carrerai (1), Ps. davisi (6) and Ps. hirsutus hirsutus (1). The samples were used to amplify a $358 \mathrm{bp}$ fragment of the cytb gene. The resultant sequences were compared with the GenBank sequences, leading to the identification of three vertebrates with $98-100 \%$ similarity: Bos taurus, Homo sapiens and Tamandua tetradactyla (Table II).

\section{DISCUSSION}

The epidemiological pattern of leishmaniasis in RO is characterised by a zoonotic or sylvatic transmission cycle in which humans might acquire infection via exposure to sandflies in the Amazon rainforest. . $^{(5,13,16)}$

A total of 73 species were registered in this study, which demonstrated a higher level of species richness than the previous surveys conducted in RO. ${ }^{(7,13,25)}$ Sand- 
TABLE I

Sandfly composition, exponential of Shannon entropy index $(q=1)$ and inverse of Simpson concentration index $(q=2)$ with its confidence intervals (CI) based on a bootstrap method of 1,000 replications for three environments from the state of Rondônia, Brazil

\begin{tabular}{|c|c|c|c|c|c|}
\hline \multirow[b]{2}{*}{ Species } & CUN & FE & PE & \multirow[b]{2}{*}{ Total } & \multirow[b]{2}{*}{$\%$} \\
\hline & & Total $(+/ ð)$ & & & \\
\hline Bichromomyia flaviscutellata (Mangabeira, 1942) ${ }^{a}$ & $65(40 / 25)$ & $65(23 / 42)$ & $9(6 / 3)$ & 139 & 1.46 \\
\hline Bichromomyia olmeca nociva (Young \& Arias, 1970) & - & - & $1(1 / 0)$ & 1 & 0.01 \\
\hline Brumptomyia brumpti (Larrousse, 1920) ${ }^{a}$ & $14(2 / 12)$ & $9(5 / 4)$ & $10(3 / 7)$ & 33 & 0.35 \\
\hline Brumptomyia mesai Sherlock, 1962 & $1(0 / 1)$ & - & - & 1 & 0.01 \\
\hline Brumptomyia pintoi (Costa Lima, 1932) & $1(0 / 1)$ & - & - & 1 & 0.01 \\
\hline Evandromyia bacula (Martins, Falcão \& Silva, 1965) ${ }^{a}$ & $9(6 / 3)$ & $2(1 / 1)$ & $2(1 / 1)$ & 13 & 0.14 \\
\hline Evandromyia georgii (Freitas \& Barrett, 2002) ${ }^{a}$ & $22(19 / 3)$ & $16(12 / 4)$ & $5(3 / 2)$ & 43 & 0.45 \\
\hline Evandromyia infraspinosa (Mangabeira, 1941) & $7(5 / 2)$ & - & - & 7 & 0.07 \\
\hline Evandromyia lenti (Mangabeira, 1938) & - & $2(1 / 1)$ & $2(1 / 1)$ & 4 & 0.04 \\
\hline Evandromyia piperiformis Godoy, Cunha \& Galati, 2017 & - & - & $1(0 / 1)$ & 1 & 0.01 \\
\hline Evandromyia saulensis (Floch \& Abonnenc, 1944) ${ }^{a}$ & $62(51 / 11)$ & $18(13 / 5)$ & $14(11 / 3)$ & 94 & 0.99 \\
\hline Evandromyia tarapacaensis (Le Pont, Torrez-Espejo \& Galati, 1997) & $2(0 / 2)$ & $2(0 / 2)$ & - & 4 & 0.04 \\
\hline Evandromyia termitophila (Martins, Falcão \& Silva, 1964) ${ }^{a}$ & $2(0 / 2)$ & $4(2 / 2)$ & $1(1 / 0)$ & 7 & 0.07 \\
\hline Evandromyia walkeri (Newstead, 1941) ${ }^{a}$ & $29(27 / 2)$ & $4(3 / 1)$ & $46(15 / 31)$ & 79 & 0.83 \\
\hline Evandromyia wilsoni (Dasmasceno \& Causey, 1945) & $17(4 / 13)$ & $3(1 / 2)$ & - & 20 & 0.21 \\
\hline Lutzomyia evangelistai Martins \& Fraiha, 1971 & $2(0 / 2)$ & - & - & 2 & 0.02 \\
\hline Lutzomyia marinkellei Young, 1979 & $2(0 / 2)$ & - & - & 2 & 0.02 \\
\hline Lutzomyia sherlocki Martins, Silva \& Falcão, $1971^{a}$ & $72(47 / 25)$ & $35(23 / 12)$ & $10(8 / 2)$ & 117 & 1.23 \\
\hline Martinsmyia waltoni (Arias, Freitas \& Barrett, 1984) & - & $1(0 / 1)$ & - & 1 & 0.01 \\
\hline Micropygomyia rorotaensis (Floch \& Abonnenc, 1944) ${ }^{a}$ & $11(1 / 10)$ & $6(1 / 5)$ & $5(2 / 3)$ & 22 & 0.23 \\
\hline Micropygomyia trinidadensis (Newstead, 1922) & - & $5(3 / 2)$ & $1(1 / 0)$ & 6 & 0.06 \\
\hline Micropygomyia villelai (Mangabeira, 1942) ${ }^{a}$ & $6(6 / 0)$ & $6(5 / 1)$ & $4(4 / 0)$ & 16 & 0.17 \\
\hline Migonemyia migonei (França, 1920) ${ }^{a}$ & $8(3 / 5)$ & $1(1 / 0)$ & $1(0 / 1)$ & 10 & 0.10 \\
\hline Nyssomyia anduzei (Rozeboom, 1942) & $26(17 / 9)$ & $1(0 / 1)$ & - & 27 & 0.28 \\
\hline Nyssomyia antunesi $\left(\right.$ Coutinho, 1939) ${ }^{a}$ & $650^{b}(447 / 203)$ & $234^{b}(169 / 65)$ & $513^{b}(235 / 278)$ & 1,397 & 14.65 \\
\hline Nyssomyia delsionatali Galati \& Galvis, 2012) ${ }^{a}$ & $1(0 / 1)$ & $1(0 / 1)$ & $5(0 / 5)$ & 7 & 0.07 \\
\hline Nyssomyia richardwardi (Ready \& Fraiha, 1981) & $123(97 / 26)$ & $12(11 / 1)$ & - & 135 & 1.42 \\
\hline Nyssomyia shawi (Fraiha, Ward \& Ready, 1981) & $14(9 / 5)$ & $1(1 / 0)$ & - & 15 & 0.16 \\
\hline Nyssomyia umbratilis (Ward \& Faiha, 1977) & $95(74 / 21)$ & $11(9 / 2)$ & - & 106 & 1.11 \\
\hline Nyssomyia whitmani (Antunes \& Coutinho, 1939) ${ }^{a}$ & $162^{b}(57 / 105)$ & $106^{b}(44 / 62)$ & $2(2 / 0)$ & 270 & 2.83 \\
\hline Nyssomyia yuilli yuilli (Young \& Porter, 1972) ${ }^{a}$ & $70(69 / 1)$ & $12(12 / 0)$ & $61(25 / 36)$ & 143 & 1.50 \\
\hline Pintomyia nevesi (Damasceno \& Arouck, 1956) ${ }^{a}$ & $21(18 / 3)$ & $18(16 / 2)$ & $7(3 / 4)$ & 46 & 0.48 \\
\hline Pintomyia serrana $\left(\right.$ Damasceno \& Arouck, 1949) ${ }^{a}$ & $6(3 / 3)$ & $13(7 / 6)$ & $2(2 / 0)$ & 21 & 0.22 \\
\hline Pintomyia sp. & $1(0 / 1)$ & - & - & 1 & 0.01 \\
\hline Pressatia calcarata (Martins \& Silva, 1964) & $1(0 / 1)$ & - & - & 1 & 0.01 \\
\hline Pressatia triacantha (Mangabeira, 1942) & $6(0 / 6)$ & - & - & 6 & 0.06 \\
\hline Psathyromyia aragaoi $(\text { Costa Lima, } 1932)^{a}$ & $36(16 / 20)$ & $8(4 / 4)$ & $2(1 / 1)$ & 46 & 0.48 \\
\hline Psathyromyia b. barretoi (Mangabeira, 1942) & $1(0 / 1)$ & $1(1 / 0)$ & - & 2 & 0.02 \\
\hline Psathyromyia campbelli (Damasceno, Causey \& Arouck, 1945) & $1(1 / 0)$ & $2(1 / 1)$ & - & 3 & 0.03 \\
\hline Psathyromyia coutinhoi (Mangabeira, 1942) & $1(0 / 1)$ & - & - & 1 & 0.01 \\
\hline Psathyromyia dendrophyla $\left(\right.$ Mangabeira, 1942) ${ }^{a}$ & $11(5 / 6)$ & $11(5 / 6)$ & $4(3 / 1)$ & 26 & 0.27 \\
\hline Psathyromyia dreisbachi $(\text { Causey \& Damasceno, } 1945)^{a}$ & $15(5 / 8)$ & $12(11 / 1)$ & $18(18 / 0)$ & 43 & 0.45 \\
\hline Psathyromyia elizabethdorvalae Brilhante, Sábio \& Galati, 2017 & $2(0 / 2)$ & $1(0 / 1)$ & - & 3 & 0.03 \\
\hline Psathyromyia hermanlenti (Martins, Silva \& Falcão, 1970) ${ }^{a}$ & $7(7 / 0)$ & $18(2 / 16)$ & $21(11 / 10)$ & 46 & 0.48 \\
\hline Psathyromyia lutziana $\left(\right.$ Costa Lima, 1932) ${ }^{a}$ & $6(3 / 3)$ & $5(3 / 2)$ & $2(2 / 0)$ & 13 & 0.14 \\
\hline
\end{tabular}




\begin{tabular}{|c|c|c|c|c|c|}
\hline \multirow[b]{2}{*}{ Species } & CUN & $\mathrm{FE}$ & $\mathrm{PE}$ & \multirow[b]{2}{*}{ Total } & \multirow[b]{2}{*}{$\%$} \\
\hline & & Total $(Q / \delta)$ & & & \\
\hline Psathyromyia runoides (Fairchild \& Hertig, 1943) & - & $6(1 / 5)$ & $9(3 / 6)$ & 15 & 0.16 \\
\hline Psychodopygus amazonensis (Root, 1934) ${ }^{a}$ & $5(5 / 17)$ & $3(3 / 1)$ & $1(1 / 0)$ & 27 & 0.28 \\
\hline Psychodopygus ayrozai (Barretto \& Coutinho, 1940) ${ }^{a}$ & $10(10 / 0)$ & $4(1 / 3)$ & $2(1 / 1)$ & 16 & 0.17 \\
\hline Psychodopygus bispinosus (Fairchild \& Hertig, 1951) & $6(6 / 0)$ & - & - & 6 & 0.06 \\
\hline Psychodopygus c. carrerai (Barretto, 1946) ${ }^{a}$ & $376^{b}(103 / 273)$ & $57(26 / 31)$ & $23(8 / 15)$ & 456 & 4.78 \\
\hline Psychodopygus chagasi $(\text { Costa Lima, } 1941)^{a}$ & $150^{b}(118 / 32)$ & $14(12 / 2)$ & $4(3 / 1)$ & 168 & 1.76 \\
\hline Psychodopygus claustrei (Root, 1934) ${ }^{a}$ & $114(24 / 90)$ & $42(7 / 35)$ & $9(2 / 7)$ & 165 & 1.73 \\
\hline Psychodopygus complexus (Mangabeira, 1941) ${ }^{a}$ & $240^{b}(165 / 75)$ & $14(0 / 14)$ & $9(0 / 9)$ & 263 & 2.76 \\
\hline Psychodopygus davisi (Root, 1934) ${ }^{a}$ & $715^{b}(442 / 273)$ & $671^{b}(239 / 432)$ & $355^{b}(138 / 217)$ & 1,741 & 18.26 \\
\hline Psychodopygus geniculatus (Mangabeira, 1941) ${ }^{a}$ & $121^{b}(108 / 13)$ & $21(15 / 6)$ & $9(4 / 5)$ & 151 & 1.58 \\
\hline Psychodopygus h. hirsutus (Mangabeira, 1942) ${ }^{a}$ & $57(49 / 8)$ & $107^{b}(45 / 52)$ & $38(20 / 18)$ & 202 & 2.12 \\
\hline Psychodopygus lainsoni Fraiha \& Ward, $1974^{a}$ & $95(56 / 39)$ & $7(5 / 2)$ & $2(0 / 2)$ & 104 & 1.09 \\
\hline Psychodopygus leonidasdeanei (Fraiha, Ryan, Ward, Lainson \& Shaw, 1986) & $115^{b}(97 / 18)$ & - & - & 115 & 1.21 \\
\hline Psychodopygus llanosmartinsi (Fraiha \& Ward, 1980) ${ }^{a}$ & $40(26 / 14)$ & $3(2 / 1)$ & $3(1 / 2)$ & 46 & 0.48 \\
\hline Psychodopygus paraensis (Costa Lima, 1941) & $19(8 / 11)$ & $6(1 / 5)$ & - & 25 & 0.26 \\
\hline Psychodopygus yucumensis (Le Pont, Caillard, Tibayrenc \& Desjeux, 1986) ${ }^{a}$ & $4(1 / 3)$ & - & $2(0 / 2)$ & 6 & 0.06 \\
\hline Sciopemyia fluviatilis (floch \& Abonnenc, 1944) ${ }^{a}$ & $3(3 / 0)$ & $3(3 / 0)$ & $1(0 / 1)$ & 7 & 0.07 \\
\hline Sciopemyia servulolimai (Damasceno \& Causey, 1945) & $10(5 / 5)$ & $7(4 / 3)$ & - & 17 & 0.18 \\
\hline Sciopemyia sordellii (Shannon \& Del Ponte, 1927) ${ }^{a}$ & $94(50 / 44)$ & $20(12 / 8)$ & $14(6 / 8)$ & 128 & 1.34 \\
\hline Trichophoromyia auraensis (Mangabeira, 1942) ${ }^{a}$ & $1,261^{b}(9 / 1252)$ & $22(2 / 20)$ & $12(0 / 12)$ & 1,295 & 13.58 \\
\hline Trichophoromyia clitella (Young \& Pérez, 1944) ${ }^{a}$ & $12^{b}(0 / 122)$ & $30(1 / 29)$ & $22(0 / 22)$ & 174 & 1.82 \\
\hline Trichophoromyia flochi (Abonnenc \& Chassignet, 1948) ${ }^{a}$ & $19(0 / 19)$ & $57(0 / 57)$ & $24(0 / 24)$ & 100 & 1.05 \\
\hline Trichophoromyia readyi (Ryan, 1986) & $4(0 / 4)$ & - & - & 4 & 0.04 \\
\hline Trichophoromyia ruifreitasi Oliveira, Teles, Medeiros, Camargo \& Pessoa, 2016 & $2(0 / 2)$ & - & - & 2 & 0.02 \\
\hline Trichophoromyia sp. & $49(48 / 1)$ & - & - & 49 & 0.51 \\
\hline Trichophoromyia ubiquitalis (Mangabeira, 1942) ${ }^{a}$ & $525^{b}(62 / 463)$ & $352^{b}(93 / 259)$ & $166^{b}(35 / 131)$ & 1.043 & 10.94 \\
\hline Trichopygomyia dasypodogeton (Castro, 1939) ${ }^{a}$ & $107(1 / 106)$ & $3(0 / 3)$ & $2(0 / 2)$ & 112 & 1.17 \\
\hline Trichopygomyia rondoniensis (Martins, Falcão \& Silva) & $6(0 / 6)$ & $1(0 / 1)$ & - & 7 & 0.07 \\
\hline Trichopygomyia sp. & $69(69 / 0)$ & - & - & 69 & 0.72 \\
\hline Viannamyia caprina (Osorno-Mesa, Moralez \& Osorno, 1972) & $11(11 / 0)$ & $12(9 / 3)$ & - & 23 & 0.24 \\
\hline Viannamyia tuberculata (Mangabeira, 1941) ${ }^{a}$ & $15(15 / 0)$ & $2(2 / 0)$ & $1(1 / 0)$ & 18 & 0.19 \\
\hline Total & $5,967(2,530 / 3,437)$ & $2,111(973 / 1,238)$ & $1,457(586 / 871)$ & 9,535 & 100 \\
\hline Sample coverage $(\%)$ & 99 & 99 & 99 & - & - \\
\hline Exponential of Shannon entropy index (CI95\%) & $16.6(16.5-17.7)$ & $16.3(15.9-17.4)$ & $8.3(8.0-8.3)$ & - & - \\
\hline Inverse of Simpson concentration index (CI95\%) & $8.2(8.2-8.7)$ & $8.5(8.5-9.3)$ & $3.6(3.6-4.0)$ & - & - \\
\hline
\end{tabular}

$a$ : species present in all environments evaluated; $b$ : abundant species in the environment. CUN: Conservation unit; FE: Forest Edge; PE: Peridomicile.

fly diversity was the highest in protected environments. The CUN environment exhibited the highest levels of species richness was found in the CUN environment, followed by the FE and PE environments; these findings corroborate those of the previous studies conducted in the Amazon region. ${ }^{(4,21,26,27)}$ Although sampling methods differed between environments, it was still possible to perform reliable diversity comparisons because the sample coverage was $99 \%$ in each environment.

Our data demonstrated that sandflies might serve as biodiversity indicators. The species richness was reduced by 10 species in the FE environments and by 21 species in the PE environments relative to the CUN environments. This indicates that the reduction of forests to small fragments affects sandfly composition primarily by eliminating the species that occur in minor abundance (rare species). ${ }^{(26,27)}$

The sandfly species Ny. antunesi, Ps. davisi and Th. ubiquitalis were abundant in all environments. Humans generally come in close proximity to forest fragments while engaged in agriculture or activities like hunting and fishing. These activities increase the risk of exposure to Leishmania vectors in possible transmission foci, ${ }^{(25,28)}$ and that risk is exacerbated by the abundance of Ny. antunesi, Ps. davisi and Th. ubiquitalis in the areas we studied. One pool of Ps. davisi tested positive for Le. (Vi.) braziliensis DNA in the municipality of Monte Negro. This finding is significant because Le. brazilien- 


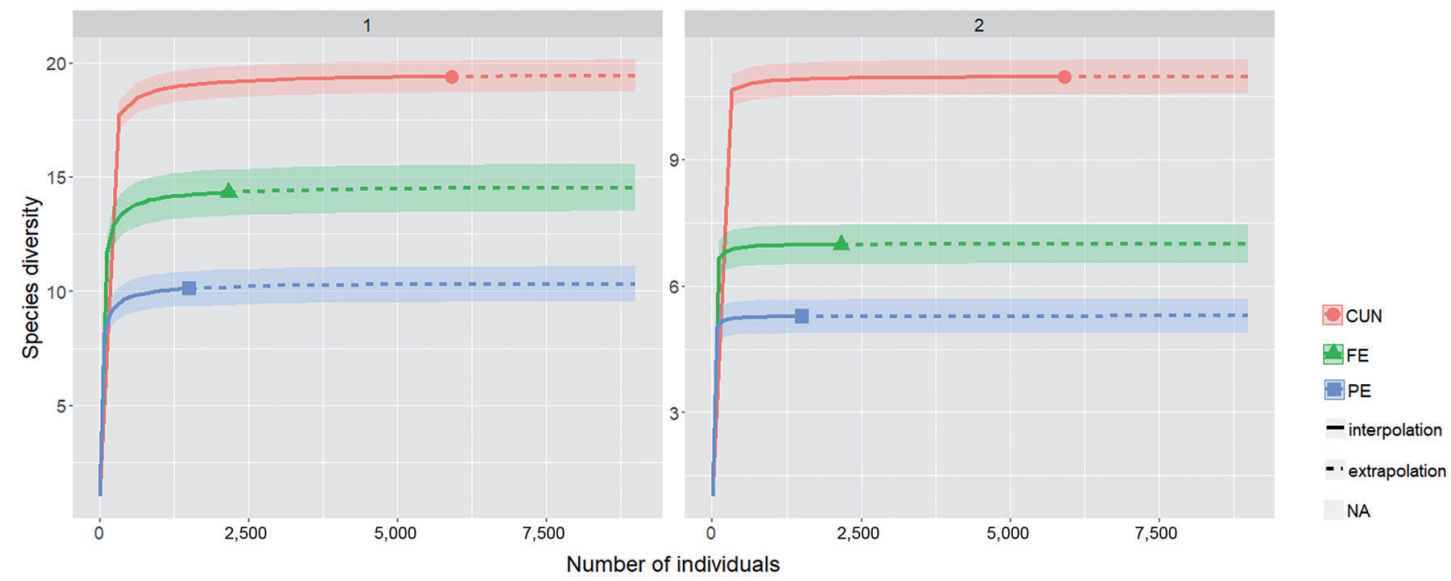

Fig. 2: index diversities based on Hill numbers of the sandfly fauna collected in three environments in the state of Rondônia, Brazil. CUN: Conservation Unit; FE: Forest Edge; PE: Peridomicile.
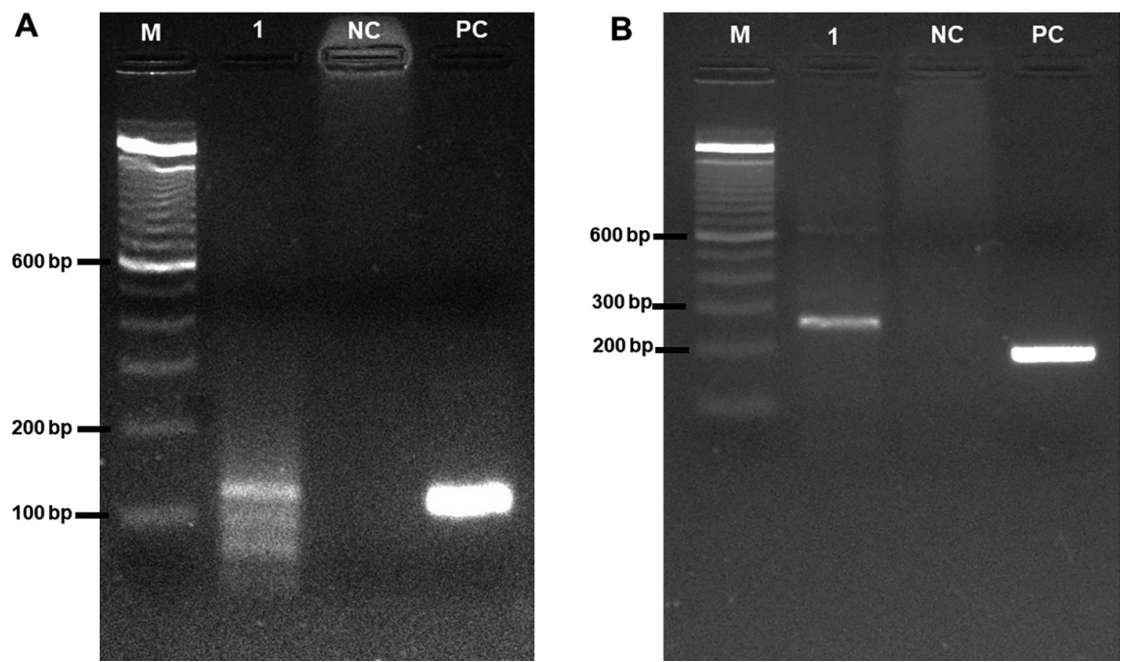

Fig. 3: natural infection of sandfly. A: amplified fragment of $120 \mathrm{bp}$ from the $k$ DNA region of the kinetoplast Leishmania species; B: the DNA extracted from the Psychodopygus davisi blood sample was subjected to polymerase chain reaction (PCR), which led to the amplification of a 240 bp hsp70 fragment. The PCR products were subjected to $1.5 \%$ agarose gel electrophoresis and stained with $1 \mu \mathrm{L}$ of GelRed ${ }^{\circledR}$ solution. 1 : Ps. davisi sample; M: molecular Maker; NC: negative control (water); PC: positive control Leishmania amazonensis reference strain IOC L0575 (IFLA/BR/1967/PH8).

sis is responsible for $50 \%$ of human CL cases in the rural population of Monte Negro. ${ }^{(25,28)}$ Ps. davisi is an abundant species in this region, ${ }^{(25)}$ as well as in other parts of $\mathrm{RO},{ }^{(4,7,13)}$ and $P$ s. davisi individuals have been previously found to be infected with Le. (Vi.) braziliensis ${ }^{(5)}$ and Le. (Vi.) naiffi. ${ }^{(13)}$ The discovery of this infection in the current study further supports the evidence that Ps. davisi might act as a vector in RO.

Furthermore, Ny. antunesi and Th. ubiquitalis might act as vectors in RO. Both species were found in high abundance in this as well as other studies conducted throughout the state,,$^{(4,13,17,25)}$ and the susceptibility of these species to natural infection by Leishmania has been demonstrated in the two studies performed in Porto Velho. ${ }^{(4,17)}$ Furthermore, both species are suspected vectors in AM and MT which border RO. ${ }^{(21,29)}$

Other abundant species found in RO included $N y$. whitmani, Ps. carrerai carrerai, Ps. complexus, Ps. hirsutus hirsutus and Th. auraensis. Ny. whitmani has already been recorded in abundance in $\mathrm{RO}^{(13,25)} \mathrm{Ny}$. whitmani and Ps. hirsutus hirsutus were found in high abundance in the FE environments, suggesting that these species are confined to degraded environments; both species have been associated with dense forest environments ${ }^{(13,25)}$ and with environments impacted by anthropic activities. ${ }^{(25,30)}$ Neither species has been found infected with Leishmania in RO; ${ }^{(4,5,7,17)}$ however, both species are putative vectors in the Amazon region because they have been found infected with Leishmania in PA. ${ }^{(6)}$

In RO, Ps. carrerai carrerai occurs mainly in dense forest environments. ${ }^{(6,7,13)}$ Only three studies conducted in central RO have demonstrated the predominance of Ps. carrerai carrerai and Ps. complexus in this region. ${ }^{(13,25)}$ PS. carrerai carrerai has been found to carry promastigote flagellates identified as Le. (Vi.) braziliensis ${ }^{(5)}$ and carrying Leishmania DNA. ${ }^{(7)}$ 
TABLE II

Vertebrate species identified from engorged sandfly females collected Forest Edge (FE) and Peridomicile (PE) environments in the state of Rondônia, Brazil

\begin{tabular}{|c|c|c|c|c|c|c|c|}
\hline Sandfly species & Blood meal & $\begin{array}{c}\text { Municipality } \\
\text { (environment) }\end{array}$ & $\begin{array}{l}\text { Accession } \\
\text { code }\end{array}$ & $\begin{array}{l}\text { Identity } \\
(\%)\end{array}$ & $\begin{array}{l}\text { Total score } \\
\text { (n) }\end{array}$ & $\begin{array}{c}\text { Query cover } \\
(\%)\end{array}$ & E-value \\
\hline Nyssomyia antunesi & Tamandua tetradactyla & Porto Velho (FE) & KT818552.1 & 98.14 & 562 & 100 & $3 \mathrm{E}-156$ \\
\hline Nyssomyia antunesi & Tamandua tetradactyla & Porto Velho (FE) & KT818552.1 & 99.07 & 582 & 100 & $2 \mathrm{E}-162$ \\
\hline Nyssomyia antunesi & Homo sapiens & Vilhena (PE) & KX697544.1 & 100 & 608 & 100 & $4 \mathrm{E}-170$ \\
\hline Bichromomyia flaviscutellata & Homo sapiens & Vilhena (FE) & KX697544.1 & 100 & 643 & 100 & $1 \mathrm{E}-180$ \\
\hline Psathyromyia dendrophyla & Bos taurus & Cacoal (PE) & MK028750.1 & 100 & 603 & 100 & $2 \mathrm{E}-168$ \\
\hline Psychodopygus davisi & Bos taurus & Cacoal (PE) & MK028750.1 & 99.66 & 538 & 100 & $4 \mathrm{E}-149$ \\
\hline Psychodopygus davisi & Bos taurus & Cacoal (PE) & MK028750.1 & 100 & 597 & 100 & 7E-167 \\
\hline Psychodopygus davisi & Bos taurus & Cacoal (PE) & MK028750.1 & 100 & 595 & 100 & $3 \mathrm{E}-166$ \\
\hline Psathyromyia dendrophyla & Bos taurus & Cacoal (PE) & MK028750.1 & 99.62 & 481 & 100 & $6 \mathrm{E}-132$ \\
\hline Psychodopygus davisi & Bos taurus & Cacoal (PE) & EU365345.1 & 99.42 & 625 & 100 & $4 \mathrm{E}-175$ \\
\hline Psychodopygus davisi & Bos taurus & Cacoal (PE) & MK028750.1 & 100 & 603 & 100 & $2 \mathrm{E}-168$ \\
\hline Psychodopygus h. hirsutus & Bos taurus & Cacoal (PE) & MK028750.1 & 99.66 & 542 & 100 & $3 \mathrm{E}-150$ \\
\hline Psychodopygus c. carrerai & Bos taurus & Cacoal (FE) & EU365345.1 & 99.05 & 566 & 100 & $2 \mathrm{E}-157$ \\
\hline Psychodopygus davisi & Homo sapiens & Ji-Paraná (FE) & KX697544.1 & 100 & 647 & 100 & 0.0 \\
\hline Nyssomyia antunesi & Homo sapiens & Ji-Paraná (PE) & KX697544.1 & 100 & 425 & 100 & $3 \mathrm{E}-115$ \\
\hline
\end{tabular}

Trichophoromyia auraensis is abundant primarily in the municipalities of Guajará-Mirim and Porto Velho and in central $\mathrm{RO}^{(13,17)}$ where Leishmania DNA has been detected in females. ${ }^{(7,17)}$ In our study, Th. auraensis was found abundant only in the CUN environments; however, the natural infection of Th. auraensis by Leishmania spp has been reported ${ }^{(30)}$ and Th. auraensis has been found in abundance in both FE and PE environments in $\mathrm{AC}$ of western Brazil. ${ }^{(19,30)}$

In our analysis of blood meal sources, blood was taken from the stomachs of 15 engorged females, and the PCR amplification of the cytb gene led to the identification of the DNA belonging to humans (H. sapiens), domestic animals (Bos taurus) and sylvatic animals such as anteaters (T. tetradactyla). The DNA belonging to Bos taurus and $H$. sapiens was present in samples from every collection made in the PE and FE environments, and this DNA was found in the blood taken from 13 female specimens belonging to the species: Bi.flaviscutellata, Ny. antunesi, Pa. dendrophyla, Ps. carrerai carrerai, Ps. davisi and Ps. hirsutus hirsutus. The T. tetradactyla DNA was present in the blood sample taken from two $N y$. antunesi females captured in the FE environments. Although 15 engorged females represents a small sample size relative to other studies, our findings are significant because previous studies targeting the cytb gene have detected the DNA of only domestic animals, such as cats, dogs, chickens, bovines, equines and pigs. ${ }^{(18-20)}$

Preserved environments showed the highest variety of blood meal sources for sandflies, and a large variety of blood meal sources guarantees the maintenance of the gonotrophic cycle. ${ }^{(12)}$ Anteaters (Tamandua spp) act as possible reservoirs for some Leishmania species, such as Le. (Vi.) guyanensis, ${ }^{(12)}$ and in degraded natural habitats, the scarcity of sylvatic reservoirs, such as ant- eaters, might cause sandflies to migrate to the PE environments to find new blood meal sources. ${ }^{(4,18,19,27)}$ In our study, the PE collection points were close to small forest fragments, and the availability of blood meal sources in the form of domestic animals, such as Bos taurus, might have attracted sandflies from the forest fragments.

Few studies have examined the importance of blood meal sources in the Brazilian Amazon. Recently, in the municipality of Rio Branco (AC), the blood collected from the intestinal contents of the two specimens of Ps. davisi was subjected to PCR targeting the cytb gene; this led to the identification of Gallus gallus as a blood meal source. ${ }^{(19)}$ The current study improves our knowledge of blood meal sources by demonstrating that vectors, such as Ny. antunesi and Ps. davisi, feed on humans and bovines in the PE environments and feed on sylvatic animals, such as anteaters in the FE environments.

Our study largely corroborates the findings of previous studies concerned with the transmission cycle of leishmaniasis in RO. However, the fact that sandflies are using humans and domestic animals as blood meal sources indicates that the transmission profile might be changing in the PE environments. These findings can be used to enhance the epidemiological surveillance of leishmaniasis in RO.

\section{ACKNOWLEDGEMENTS}

To Conselho Nacional de Desenvolvimento Científico e Tecnológico (CNPq) (chamada MCTI/CNPq/FNDCT Ação Transversal - Redes Regionais de Pesquisa em Ecossistemas, Biodiversidade e Biotecnologia $n^{\circ}$ 79/2013). This study was also financed in part by the Coordenação de Aperfeiçoamento de Pessoal de Nível Superior (Capes, Brazil, financial code 001). To the Program for Technological Development in Tools for Health-PDTIS/FIOCRUZ, for the use of its facilities. 


\section{AUTHORS' CONTRIBUTIONS}

AMPJ, GEMF and JFM obtained the funding, conceived ideas and design of the study; AMPJ, ABNS, MSS, TSC and PFMP performed the sandfly collection and identification and molecular biology procedures; AMPJ, GEMF and JFM drafted the manuscript. All authors approved the final version of the manuscript.

\section{REFERENCES}

1. Ready PD. Biology of phlebotomine sand flies as vectors of disease agents. Annu Rev Entomol. 2013; 58(1): 227-50.

2. Galati EAB. Phlebotominae (Diptera, Psychodidae): classification, morphology and terminology of adults and identification of American taxa. In: Rangel EF, Shaw JJ. Brazilian sand flies. Gewerbestrasse: Springer; 2018. p. 9-212.

3. Rangel EF, Lainson R, Carvalho BM, Costa SM, Shaw JJ. Sand fly vectors of American cutaneous leishmaniasis in Brazil. In: Rangel EF, Shaw JJ. Brazilian sand flies. Gewerbestrasse: Springer; 2018. p. $341-80$

4. Resadore F, Pereira Júnior AM, Carvalho LPC, Santos APA, Teles CBG, Medeiros JF. Phlebotomine sand fly composition (Diptera: Psychodidae) and putative vectors of American cutaneous leishmaniasis in Porto Velho municipality, Western Amazon, Brazil. J Med Entomol. 2017; 54(3): 798-803.

5. Grimaldi G, Momen H, Naiff RD, McMahon-Pratt D, Barrett TV. Characterization and classification of leishmanial parasites from humans, wild mammals, and sand flies in the Amazon Region of Brazil. Am J Trop Med Hyg. 1991; 44(6): 645-61.

6. Souza AAA, Santos TV, Jennings YLL, Ishikawa EAY, Barata IR, Silva MGS, et al. Natural Leishmania (Viannia) spp infections in phlebotomine sand flies (Diptera: Psychodidae) from the Brazilian Amazon region reveal new putative transmission cycles of American cutaneous leishmaniasis. Parasite. 2016; 23(2016): 22.

7. Resadore F, Pereira Júnior AM, Paulo PFM, Gil LHS, Rodrigues MMS, Araújo MS, et al. Composition and vertical stratification of phlebotomine sand fly fauna and the molecular detection of Leishmania in forested areas in Rondônia state municipalities, Western Amazon, Brazil. Vector-Borne Zoonotic Dis. 2019; 19(5): 347-57.

8. Santos SO, Arias J, Ribeiro AA, Hoffmann M, Freitas RA, Malacco MA. Incrimination of Lutzomyia cruzi as a vector of American visceral leishmaniasis. Med Vet Entomol. 1998; 12(3): 315-7.

9. Guimarães VCFV, Pruzinova K, Sadlova J, Volfova V, Myskova J, Filho SPB, et al. Lutzomyia migonei is a permissive vector competent for Leishmania infantum. Parasit Vectors. 2016; 9: 159.

10. Galvis-Ovallos F, Silva MD, Bispo GBS, Oliveira AG, Neto JRG, Malafronte RS, et al. Canine visceral leishmaniasis in the metropolitan area of São Paulo: Pintomyia fischeri as potential vector of Leishmania infantum. Parasite. 2017; 24: 2.

11. Ministério da Saúde. Departamento de Informática do Sistema Único de Saúde (DATASUS) (BR). Sistema de informações de agravos de notificação. Epidemiológicas e Morbidade. Leishmaniose tegumentar americana [Internet]. Brasília, DF: DATASUS; 2018 [updated 2018, cited 2018 Jan 20]. Available from: http://www2.datasus.gov.br/DATASUS/index.php?area $=0203 \&$ $\mathrm{id}=29892200 \& \mathrm{VObj}=\mathrm{http}: / /$ tabnet.datasus.gov.br/cgi/deftohtm. exe?sinannet/cnv/lta/.

12. Lainson R, Shaw JJ, Póvoa M. The importance of edentates (sloths and anteaters) as primary reservoirs of Leishmania braziliensis guyanensis, causative agent of "pianbois" in north Brazil. Trans R Soc Trop Med Hyg. 1981; 75(4): 611-2.
13. Gil LHS, Basano SA, Souza AA, Silva MGS, Barata IR, Ishikawa EA, et al. Recent observations on the sand fly (Diptera: Psychodidae) fauna of the state of Rondônia, Western Amazônia, Brazil: the importance of Psychdopygus davisi as a vector of zoonotic cutaneous leishmaniasis. Mem Inst Oswaldo Cruz. 2003; 98(6): 751-5.

14. Confalonieri UEC, Margonari C, Quintão AF. Environmental change and the dynamics of parasitic diseases in the Amazon. Acta Trop. 2014; 129: 33-41.

15. Silva JC, Mattos CB, Felipin KP, Silva HPJ, Cantanhêde LM, Porrozzi R, et al. First autochthonous case of canine vicsceral leishmaniasis in Rondônia, Brazil, a region with no history of visceral leishmaniasis. Rev Soc Bras Med Trop. 2018; 51(5): 712-5.

16. Cantanhêde LM, Silva Júnior CF da, Ito MM, Felipin KP, Nicolete $\mathrm{R}$, Salcedo JMV, et al. Further evidence of an association between the presence of Leishmania RNA virus 1 and the mucosal manifestations in tegumentary leishmaniasis patients. PLoS Negl Trop Dis. 2015; 9(9): e0004079.

17. Ogawa GM, Pereira Júnior AM, Resadore F, Ferreira RGM, Medeiros JF, Camargo LMA. Sandfly fauna (Diptera: Psychodidae) from caves in the state of Rondônia, Brazil. Rev Bras Parasitol Veterinária. 2016; 25(1): 61-8.

18. Quaresma PF, Carvalho GML, Ramos MCNF, Andrade Filho JD. Natural Leishmania sp. reservoirs and phlebotomine sandfly food source identification in Ibitipoca State Park, Minas Gerais, Brazil. Mem Inst Oswaldo Cruz. 2012; 107(4): 480-5.

19. Ávila MM, Brilhante AF, Souza CF, Bevilacqua PD, Galati EAB, Brazil RP. Ecology, feeding and natural infection by Leishmania spp of phlebotomine sand flies in an area of high incidence of American tegumentary leishmaniasis in the municipality of Rio Branco, Acre, Brazil. Parasit Vectors. 2018; 11(1): 64.

20. Carvalho GML, Rêgo FD, Tanure A, Silva ACP, Dias TA, Paz GF, et al. Bloodmeal identification in field-collected sand flies from Casa Branca, Brazil, using the cytochrome $b$ PCR method. J Med Entomol. 2017; 54(4): 1049-54.

21. Pereira Júnior AM, Teles CBG, Santos APA, Rodrigues MS, Marialva EF, Pessoa FAC, et al. Ecological aspects and molecular detection of Leishmania DNA Ross (Kinetoplastida: Trypanosomatidae) in phlebotomine sandflies (Diptera: Psychodidae) in terra firme and várzea environments in the Middle Solimões Region, Amazonas state, Brazil. Parasit Vectors. 2015; 8: 180.

22. Sambrook J, Russell D. Isolament and quantification of DNA. In: Inglis J, Boyle A, Gann A. Molecular Cloning. A laboratory manual molecular cloning. A laboratory manual. New York: Cold Spring Harbor Laboratory Press; 2012. p. 1-78.

23. Boakye DA, Tang J, Truc P, Merriweather A, Unnasch TR. Identification of bloodmeals in haematophagous Diptera by cytochrome B heteroduplex analysis. Med Vet Entomol. 1999; 13(3): 282-7.

24. Ewing B, Green P. Base-calling of automated sequencer traces using phred. II. Error probabilities. Genome Res. 1998; 8(3): 186-94.

25. Teles CBG, Basano SA, Zagonel-Oliveira M, Campos JJ, Oliveira AFJ, Freitas RA, et al. Epidemiological aspects of American cutaneous leishmaniasis and phlebotomine sandfly population, in the municipality of Monte Negro, state of Rondônia, Brazil. Rev Soc Bras Med Trop. 2013; 46(1): 60-6.

26. Pessoa FAC, Medeiros JF, Barrett TV. Effects of timber harvest on phlebotomine sand flies (Diptera: Psychodidae) in a production forest: abundance of species on tree trunks and prevalence of trypanosomatids. Mem Inst Oswaldo Cruz. 2007; 102(5): 593-9.

27. Ramos WR, Medeiros JF, Julião GR, Ríos-Velásquez CM, Mar- 
ialva EF, Desmoulière SJM, et al. Anthropic effects on sand fly (Diptera: Psychodidae) abundance and diversity in an Amazonian rural settlement, Brazil. Acta Trop. 2014; 139: 44-52.

28. Shaw JJ, Faria DL, Basano SA, Corbett CE, Rodrigues CJ, Ishikawa EEA, et al. The aetiological agents of American cutaneous leishmaniasis in the municipality of Monte Negro, Rondônia state, Western Amazonia, Brazil. Ann Trop Med Parasitol. 2007; 101(8): 681-8.
29. Thies SF, Bronzoni RVM, Michalsky EM, Santos ES, Silva DJF, Dias ES, et al. Aspects on the ecology of phlebotomine sand flies and natural infection by Leishmania hertigi in the southeastern Amazon Basin of Brazil. Acta Trop. 2018; 177: 37-43.

30. Teles CBG, Santos APA, Freitas RA, Oliveira AFJ, Ogawa GM, Rodrigues MS, et al. Phlebotomine sandfly (Diptera: Psychodidae) diversity and their Leishmania DNA in a hot spot of American cutaneous leishmaniasis human cases along the Brazilian border with Peru and Bolivia. Mem Inst Oswaldo Cruz. 2016; 111(7): 423-32. 